\title{
Second virial coefficients of asymmetric top molecules
}

\author{
Paul E. S. Wormer \\ Theoretical Chemistry, IMM, Radboud University Nijmegen, Toernooiveld 1, 6525 ED Nijmegen, \\ The Netherlands
}

(Received 3 January 2005; accepted 21 February 2005; published online 6 May 2005)

\begin{abstract}
A short self-contained derivation is given for the second virial coefficient $B_{2}(T)$ of a gas consisting of identical interacting asymmetric rigid rotors. The resulting expression is correct through $\hbar^{2}$. First, the canonical partition function is derived by means of an $\hbar$ expansion of $\exp \left[-H /\left(k_{B} T\right)\right]$ due to Friedmann [Adv. Chem. Phys. 4, 225 (1962)]. The present work applies angular momentum operators and known facts from angular momentum theory. It is considerably more accessible than Friedmann's exposition, which is not based on angular momentum operators, but instead on explicit derivatives with respect to Euler angles. The partition function obtained from the $\hbar$ expansion is applied to the derivation of an expression for $B_{2}(T)$ that is identical in appearance to the expression for symmetric rotors of T Pack [J. Chem. Phys. 78, 7217 (1983)]. The final equation in this work is valid for rigid rotors of any symmetry. (C) 2005 American Institute of Physics.
\end{abstract}

[DOI: 10.1063/1.1889428]

\section{INTRODUCTION}

Second virial coefficients $B_{2}(T)$, which describe the first nonideality correction to the Boyle-Gay-Lussac (ideal gas) law $p V=R T$, depend sensitively on the pair potential of the molecules in the gas. Because virial coefficients can be measured, their ab initio computation and comparison with experimental values provide a valuable check on the accuracy of $a b$ initio computational results. See for a recent example of such comparisons Ref. 1.

About 20 years ago $\mathrm{T}$ Pack $^{2}$ published a paper on the first quantum corrections to $B_{2}(T)$. He gave a compact formula and restricted its validity specifically to symmetric tops. The reason for this restriction was that $\mathrm{T}$ Pack used a formula for the $N$-body canonical partition function derived earlier by Friedmann ${ }^{3}$ for the special case of symmetric tops. The general expression for a partition function is

$$
Q_{N}(T) \equiv \operatorname{Tr} e^{-\beta H(1,2, \ldots, N)},
$$

where $\beta=1 /\left(k_{B} T\right), \quad k_{B}$ is the Boltzmann constant, and $H(1,2, \ldots, N)$ is the Hamiltonian of a system of $N$ interacting rigid rotors. One of the purposes of this paper is to show that T Pack's equation is valid equally well for gases consisting of asymmetric tops. We will do this by deriving the first quantum correction to $Q_{N}$, or rather to $Q_{2}$. Only the case $N$ $=2$ is of importance for virial coefficients, since they may be obtained from ${ }^{4}$

$$
B_{2}(T)=V\left(\frac{1}{2}-\frac{Q_{2}(T)}{Q_{1}(T)^{2}}\right),
$$

where $V$ is the volume of the vessel containing the gas.

In this paper we will follow an idea of Friedmann ${ }^{3}$ concerning the expansion of $\exp [-\beta H]$ in orders of $\hbar$. Friedmann considered the first $\left(\hbar^{2}\right)$ quantum correction to $Q_{N}$ for systems of $N$ diatoms and $N$ symmetric tops. His expansion will be applied below to $Q_{2}$ in a much simpler setting. Friedmann's approach is very complicated as he starts out from the general Laplace-Beltrami-Podolsky ${ }^{5,6}$ form of the Hamiltonian valid for arbitrary coordinate systems (even for those with nondiagonal metric tensors, such as Euler angles). The inverse of the metric tensor, its derivatives, and its determinant appear in the Hamiltonian. After a quite complicated development Friedmann specialized his general theory to diatoms and symmetric tops, both of which have metric tensors that can be found in textbooks. ${ }^{7}$ However, both types of systems can be treated very well with angular momentum theory without any explicit reference to metric tensors. The metric tensor of an asymmetric top is somewhat more complicated than that of a symmetric top and Friedmann did not consider the former case. A compact derivation of quantum corrections to $Q_{N}$ will be given that is more accessible than Friedmann's 30 odd pages. We will look at asymmetric tops using angular momentum operators instead of coordinate derivatives. The complicated Laplace-Beltrami-Podolsky Hamiltonian is replaced by the simple rigid-rotor Hamiltonian

$$
H_{\mathrm{rot}}=\frac{\mathcal{J}_{x}^{2}}{2 I_{x}}+\frac{\mathcal{J}_{y}^{2}}{2 I_{y}}+\frac{\mathcal{J}_{z}^{2}}{2 I_{z}},
$$

where $I_{a}$ is an inertia moment and $\mathcal{J}_{a}(a=x, y, z)$ is a component of the body-fixed angular momentum operator. ${ }^{8}$ The symmetric top will follow as the special case that two out of the three inertia moments are equal.

\section{ONE-BODY THEORY}

We establish notation and review a few necessary facts concerning a canonical ensemble ( $T-V-N$ constant) of systems of $N$ identical noninteracting rigid rotors.

A rigid rotor has six degrees of freedom: the position $\mathbf{r}$ of its center of mass, and its orientation, given by the three Euler angles $\boldsymbol{\omega} \equiv\left(\omega_{1}, \omega_{2}, \omega_{3}\right)$. Thus, a rotor has the coordinates $\mathbf{x} \equiv(\mathbf{r}, \boldsymbol{\omega})$. The quantum mechanical kinetic energy of a 
rotor is a sum of a translational part $H_{\mathrm{tr}}=\mathbf{p} \cdot \mathbf{p} /(2 M)$, with $M$ the mass of the rotor, and a rotational part $H_{\text {rot }}[\mathrm{Eq}$. (3)]. The one-body partition function factorizes

$$
Q_{1}=\operatorname{Tr}\left(e^{-\beta H_{\text {tr }}}\right) \operatorname{Tr}\left(e^{-\beta H_{\text {rot }}}\right) \equiv Q_{1}^{\text {tr }} Q_{1}^{\text {rot }} .
$$

The operator $p_{a} \equiv-i \hbar \partial / \partial r_{a}(a=x, y, z)$ is Hermitian. By partial integration this can be proved, provided the resulting surface integrals vanish, which we assume to be the case. The angular momentum operators $\mathcal{J}_{x}, \mathcal{J}_{y}$, and $\mathcal{J}_{z}$ are Hermitian since they act on periodic functions of the Euler angles. Note that while $\left[p_{a}, p_{b}\right]=0(a, b=x, y, z)$, the body-fixed operators $\mathcal{J}_{a}$ satisfy the well-known ${ }^{8}$ anomalous commutation relations.

Let us consider first the necessary translational equations. The one-body partition function computed in the $\mathbf{r}$ representation is the integral over the diagonal element (Slater sum):

$$
Q_{1}^{\mathrm{tr}}=\int_{V} d \mathbf{r} W_{1}^{\mathrm{tr}}(\mathbf{r}) \quad \text { with } W_{1}^{\mathrm{tr}}(\mathbf{r})=\left\langle\mathbf{r}\left|e^{-\beta H_{\mathrm{tr}}}\right| \mathbf{r}\right\rangle
$$

We assume on the one hand that the operators $\mathbf{r}$ and $\mathbf{p}$ have a continuous spectrum, while on the other we assume that $V$ is finite. In the final result $V$ will drop out and we then take the limit of infinite $V$. As is well-known, ${ }^{9}$ the Slater sum is independent of $\mathbf{r}$ and is related to de Broglie thermal length $\Lambda$ by

$$
W_{1}^{\mathrm{tr}}=\Lambda^{-3} \equiv\left(\frac{2 \pi M k_{B} T}{h^{2}}\right)^{3 / 2}
$$

so that $Q_{1}^{\mathrm{tr}}=V \Lambda^{-3}$.

In the following section we will meet the expression $\left\langle\mathbf{r}\left|p_{a} p_{b} \exp \left(-\beta H_{\mathrm{tr}}\right)\right| \mathbf{r}\right\rangle$ bilinear in the momenta $(a, b=x, y, z)$ and a similar expression linear in $p_{a}$. Temporarily widening our view somewhat, we introduce the homogeneous polynomial $P\left(p_{x}, p_{y}, p_{z}\right)$ and consider

$$
\left\langle\mathbf{r}\left|P\left(p_{x}, p_{y}, p_{z}\right) e^{-\beta H_{\mathrm{tr}}}\right| \mathbf{r}\right\rangle .
$$

If $P$ is of odd order, this expectation value vanishes. Indeed, the $p_{a}$ 's commute mutually so that the total operator in Eq. (6) is Hermitian and the expectation value is real. Further, the operator is antisymmetric under time reversal if $P\left(p_{x}, p_{y}, p_{z}\right)$ is of odd order, so that its expectation value over a state that is even or odd under time-reversal symmetry is purely imaginary. In total the expectation value vanishes for odd-order $P$. Using the resolution of the identity in the p representation and $\langle\mathbf{r} \mid \mathbf{p}\rangle=h^{-3 / 2} \exp (i \mathbf{r} \cdot \mathbf{p})$, so that $\langle\mathbf{r} \mid \mathbf{p}\rangle\langle\mathbf{p} \mid \mathbf{r}\rangle=h^{-3}$, we find by simple integration,

$$
\frac{1}{2 M}\left\langle\mathbf{r}\left|p_{a} p_{b} e^{-\beta H_{\mathrm{tr}}}\right| \mathbf{r}\right\rangle=\delta_{a b} \frac{k_{B} T}{2} \Lambda^{-3}=\delta_{a b} \frac{k_{B} T}{2} \frac{Q^{\operatorname{tr}}}{V},
$$

where the Kronecker $\delta$ is due to reflection symmetry in $\mathbf{p}$ space. Since the translational matrix element is independent of $a=x, y, z$, we find here the equipartition of energy over the three translation degrees of freedom. The statistical averages of the energies of the three translational modes add up to the total translational energy $\frac{3}{2} k_{B} T$.

We now turn to the required rotational equations. An asymmetric top wave function and its eigenvalue are ob- tained by diagonalizing $H_{\text {rot }}$ on the space spanned by the elements (symmetric top eigenfunctions ${ }^{10}$ ) in row $M$ of the complex conjugate of a Wigner $D$ matrix. ${ }^{11}$ The result is independent of $M$. That is, the eigenfunctions belonging to the $(2 J+1)$-degenerate energy $E_{J \tau}$ are

$$
\langle\boldsymbol{\omega} \mid J M \tau\rangle=\sqrt{\frac{2 J+1}{8 \pi^{2}}} \sum_{K=-J}^{J} c_{K \tau} D_{M K}^{J}(\boldsymbol{\omega})^{*}, \quad M=-J, \ldots, J,
$$

where $D_{M K}^{J}(\boldsymbol{\omega})^{*}$ is a symmetric top eigenfunction. Invocation of the great orthogonality relation for the Wigner $D$ matrices [which constitute an irreducible matrix representation of the full rotation group $\mathrm{SO}(3)$ ] shows that the states are orthogonal on $J$ and $M$ and, being eigenstates of $H_{\text {rot }}$, also on $\tau$,

$$
\int d \boldsymbol{\omega}\left\langle J^{\prime} M^{\prime} \tau^{\prime} \mid \boldsymbol{\omega}\right\rangle\langle\boldsymbol{\omega} \mid J M \tau\rangle=\delta_{J^{\prime} J} \delta_{M^{\prime} M} \delta_{\tau^{\prime} \tau},
$$

with $d \boldsymbol{\omega}=\sin \omega_{2} d \omega_{1} d \omega_{2} d \omega_{3}$.

The Slater sum $W_{1}^{\text {rot }}=\left\langle\boldsymbol{\omega}\left|e^{-\beta H_{\text {rot }}}\right| \boldsymbol{\omega}\right\rangle$ is independent of $\boldsymbol{\omega}$. This statement can be generalized to the operator

$$
\rho \equiv P\left(\mathcal{J}_{x}, \mathcal{J}_{y}, \mathcal{J}_{z}\right) e^{-\beta H_{\text {rot }}}
$$

where $P\left(\mathcal{J}_{x}, \mathcal{J}_{y}, \mathcal{J}_{z}\right)$ is a homogeneous polynomial. Since the $\mathcal{J}_{a}$ 's do not commute mutually we cannot use the same manner of proof as for the $p_{a}$ 's. Therefore, we consider first

$$
\begin{aligned}
\sum_{M}\langle\boldsymbol{\omega} \mid J M \tau\rangle\left\langle J M \tau^{\prime} \mid \boldsymbol{\omega}\right\rangle \\
=\frac{2 J+1}{8 \pi^{2}} \sum_{K K^{\prime}} c_{K \tau} c_{K^{\prime} \tau^{\prime}}^{*} \sum_{M} D_{M K}^{J}(\boldsymbol{\omega})^{*} D_{M K^{\prime}}^{J}(\boldsymbol{\omega}) \\
=\frac{2 J+1}{8 \pi^{2}} \sum_{K} c_{K \tau} c_{K \tau^{\prime}}^{*}=\frac{2 J+1}{8 \pi^{2}} \delta_{\tau \tau^{\prime}} .
\end{aligned}
$$

The dependence on $\boldsymbol{\omega}$ has dropped out because of the homomorphism condition satisfied by the unitary $D$ matrices. Introduction of the resolution of the identity in the $J M \tau$ representation into $\langle\boldsymbol{\omega}|\rho| \boldsymbol{\omega}\rangle$ in three places, employment of the diagonality of $P\left(\mathcal{J}_{x}, \mathcal{J}_{y}, \mathcal{J}_{z}\right)$ in $J$ and $M$ and the independence of its matrix elements on $M$ (because the body-fixed angular momenta commute with the space-fixed $J_{z}$, whose eigenvalue is $M$ ), taken together with the diagonality of $\exp \left[-\beta H_{\text {rot }}\right]$ in $J, M$ and $\tau$ gives for $\langle\boldsymbol{\omega}|\rho| \boldsymbol{\omega}\rangle$ the result

$$
\begin{aligned}
\langle\boldsymbol{\omega}|\rho| \boldsymbol{\omega}\rangle= & \sum_{J M \tau \tau^{\prime} \tau^{\prime \prime}}\langle\boldsymbol{\omega} \mid J M \tau\rangle\left\langle J M \tau\left|P\left(\mathcal{J}_{x}, \mathcal{J}_{y}, \mathcal{J}_{z}\right)\right| J M \tau^{\prime}\right\rangle \\
& \times\left\langle J M \tau^{\prime}\right| e^{-\beta H_{\mathrm{rot}}\left|J M \tau^{\prime \prime}\right\rangle\left\langle J M \tau^{\prime \prime} \mid \boldsymbol{\omega}\right\rangle} \\
= & \frac{1}{8 \pi^{2}} \sum_{J M \tau}\left\langle J M \tau\left|P\left(\mathcal{J}_{x}, \mathcal{J}_{y}, \mathcal{J}_{z}\right) e^{-\beta H_{\mathrm{rot}}}\right| J M \tau\right\rangle .
\end{aligned}
$$

In particular,

$$
W_{1}^{\mathrm{rot}}=\left\langle\boldsymbol{\omega}\left|e^{-\beta H_{\mathrm{rot}}}\right| \boldsymbol{\omega}\right\rangle=\frac{Q_{1}^{\mathrm{rot}}}{8 \pi^{2}} .
$$

The trace in Eq. (10) does not necessarily vanish for polynomials $P\left(\mathcal{J}_{x}, \mathcal{J}_{y}, \mathcal{J}_{z}\right)$ of odd order. The asymmetric top Hamiltonian $H_{\text {rot }}$ belongs to the Abelian symmetry group $D_{2}$, which consists of rotations by $\pi$ around the three inertial 
axes. A rotation around the $x$ axis gives $\mathcal{J}_{y} \mapsto-\mathcal{J}_{y}$, $\mathcal{J}_{z} \mapsto-\mathcal{J}_{z}$, and $\mathcal{J}_{x} \mapsto \mathcal{J}_{x}$. The rotations around the $y$ and $z$ axis give the corresponding (cyclically permuted) maps. So, the three operators $\mathcal{J}_{x}, \mathcal{J}_{y}$, and $\mathcal{J}_{z}$ transform according to different irreducible representations (irreps) of $D_{2}{ }^{12}$ Rigid rotor functions $|J M \tau\rangle$ belong to irreps of $D_{2}$. An expectation value (bra and ket the same) of an arbitrary operator with respect to rigid rotor functions is nonvanishing if and only if the operator transforms as the totally symmetric irrep $A_{1}$. Since $\mathcal{J}_{x}$, $\mathcal{J}_{y}$, and $\mathcal{J}_{z}$ do not transform as $A_{1}$ their expectation values vanish. In second order only the squares $\mathcal{J}_{x}^{2}, \mathcal{J}_{y}^{2}$, and $\mathcal{J}_{z}^{2}$ transform as $A_{1}$, while out of all third-order monomials only the product $\mathcal{J}_{x} \mathcal{J}_{y} \mathcal{J}_{z}$ transforms as $A_{1}$. If higher than $\hbar^{2}$ quantum corrections are contemplated this latter ternary product must not be overlooked, which is easy to do because its expectation value is zero in the case of the symmetric top with its much higher symmetry $D_{\infty}$.

We will need expressions for $\left\langle\boldsymbol{\omega}\left|\mathcal{J}_{a}^{2} e^{-\beta H_{\text {rot }} \mid}\right| \boldsymbol{\omega}\right\rangle \quad(a$ $=x, y, z)$. While the corresponding expressions for $p_{a}^{2}$ [Eq. (7)] could be obtained by integration, there are no closed formulas for these expressions. Introducing the rotational temperature $\Theta_{a}=\hbar^{2} /\left(2 I_{a} k_{B}\right)$ we can write, however,

$$
\frac{\operatorname{Tr}\left(\mathcal{J}_{a}^{2} e^{\left.-\beta H_{\mathrm{rot}}\right)}\right.}{Q_{1}^{\mathrm{rot}}}=-T \hbar^{2} \frac{\partial \ln Q_{1}^{\mathrm{rot}}}{\partial \Theta_{a}}, \quad a=x, y, z,
$$

and from Eq. (10)

$$
\left\langle\boldsymbol{\omega}\left|\mathcal{J}_{a}^{2} e^{-\beta H_{\mathrm{rot}}}\right| \boldsymbol{\omega}\right\rangle=-\frac{Q_{1}^{\mathrm{rot}} T h^{2}}{2} \frac{\partial \ln Q_{1}^{\mathrm{rot}}}{\partial \Theta_{a}} .
$$

Stripp and Kirkwood ${ }^{13}$ derived an approximation for $Q_{1}^{\text {rot }}$ that consists of a sum of a classical and a first-order quantum term. The first term $Q_{1}^{\text {rot,class }}$ is an integral over phase space (premultiplied by $h^{-3}$ ), which can be computed analytically, giving

$$
Q_{1}^{\text {rot,class }}=\left(\frac{\pi T^{3}}{\Theta_{x} \Theta_{y} \Theta_{z}}\right)^{1 / 2} .
$$

The second term is the first $\left[O\left(\hbar^{2}\right)\right]$ quantum correction, which, according to Stripp and Kirkwood, ${ }^{13}$ is

$$
Q_{1}^{\text {rot,qm(1) }}=\frac{Q_{1}^{\text {rot,class }}}{12 T} \sum_{x, y, z \text { cyclic }}\left[2 \Theta_{x}-\frac{\Theta_{y} \Theta_{z}}{\Theta_{x}}\right] .
$$

Substitution of Eqs. (12) and (13) into Eq. (11) yields an expression that is valid through $\hbar^{2}$, namely,

$$
\left\langle\boldsymbol{\omega}\left|\frac{\mathcal{J}_{a}^{2}}{2 I_{a}} e^{-\beta H_{\mathrm{rot}}}\right| \boldsymbol{\omega}\right\rangle=\frac{k_{B} T Q_{1}^{\mathrm{rot}}}{8 \pi^{2}}\left(\frac{1}{2}-F_{a}(T)\right)+O\left(\hbar^{4}\right)
$$

with $F_{a}(T)$ given by $(a \neq b \neq c)$

$$
F_{a}(T)=\frac{\left[\Theta_{a}\left(\Theta_{b}-\Theta_{c}\right)\right]^{2}-\left(\Theta_{b} \Theta_{c}\right)^{2}}{P(T)},
$$

in which $P(T)$ is given as

$$
\begin{aligned}
P(T)= & -2 \Theta_{x} \Theta_{y} \Theta_{z}\left(6 T+\Theta_{x}+\Theta_{y}+\Theta_{z}\right)+\Theta_{y}^{2} \Theta_{x}^{2}+\Theta_{y}^{2} \Theta_{z}^{2} \\
& +\Theta_{z}^{2} \Theta_{x}^{2} .
\end{aligned}
$$

In the same $O\left(\hbar^{2}\right)$ approximation the quantum statistical average $\left\langle\left\langle H_{\text {rot }}\right\rangle\right\rangle$ of the rotational Hamiltonian is given by

$$
\left\langle\left\langle H_{\mathrm{rot}}\right\rangle\right\rangle=\frac{\operatorname{Tr} H_{\mathrm{rot}} e^{-\beta H_{\mathrm{rot}}}}{Q_{1}^{\text {rot }}}=k_{B} T\left(\frac{3}{2}-\sum_{a=x, y, z} F_{a}(T)\right) .
$$

In the following section we will show that it is consistent to neglect $F_{x}, F_{y}$, and $F_{z}$ in the first quantum correction of $B_{2}(T)$.

By computing $\operatorname{Tr}\left[H_{\text {rot }} \exp \left(-\beta H_{\text {rot }}\right)\right]$ and $\operatorname{Tr}[\exp (-\beta H$ rot $)]$ numerically, one can approximate the value of $\left\langle\left\langle H_{\text {rot }}\right\rangle\right\rangle$ to any precision. This makes it possible to investigate how good an approximation Eq. (16) gives. Because the $F_{a}(T)$ 's originate from a first-order quantum correction to the classical partition function, their sum gives only a first-order correction to $\left\langle\left\langle H_{\text {rot }}\right\rangle\right\rangle$. Upon recalling the definition of $H_{\text {rot }}$ in Eq. (3), we note that the operation of $\mathcal{J}_{a}$ on the symmetric top wave functions $D_{M K}^{J}(\boldsymbol{\omega})^{*}$ is well defined, and via the use of step-up(down) operators easily implemented. Thus, in the basis $D_{M K}^{J}(\boldsymbol{\omega})^{*}$ ( $J$ and $K$ running) the matrix of $H_{\text {rot }}$ can be readily calculated. This matrix is block diagonal in $J$ and independent of $M$. After diagonalization of the subblocks, yielding the eigenvalues $E_{J \tau}$, the matrices of $\exp \left(-\beta H_{\text {rot }}\right)$ and $H_{\text {rot }} \exp \left(-\beta H_{\text {rot }}\right)$ become diagonal and functions of $E_{J \tau}$, so that their contributions to $\left\langle\left\langle H_{\text {rot }}\right\rangle\right\rangle$ are easily calculated. By increasing the maximum $J$ in this procedure and monitoring convergence, the quantum statistical average of $H_{\text {rot }}$ can be computed to any required precision.

In order to illustrate the magnitude of $F_{x}, F_{y}$, and $F_{z}$ and the error that their neglect will introduce, we consider the $\mathrm{H}_{2} \mathrm{O}$ and $\mathrm{SO}_{2}$ molecules as typical examples of asymmetric tops. From $m_{\mathrm{H}}=1.0078252 \mathrm{u}, m_{\mathrm{O}}=15.9949150 \mathrm{u}, m_{\mathrm{S}}$ $=31.97207069 \mathrm{u}, \quad r_{\mathrm{OH}}=0.9572 \AA, \quad r_{\mathrm{SO}}=1.4321 \AA$, $\angle \mathrm{H}-\mathrm{O}-\mathrm{H}=104.5^{\circ}, \angle \mathrm{O}-\mathrm{S}-\mathrm{O}=119.5^{\circ}$, we obtain the following rotational temperatures (the molecules are in the $x y$ plane, the $y z$ plane is the symmetry plane):

$$
\begin{aligned}
\mathrm{H}_{2} \mathrm{O}: \Theta_{x}= & 39.455 \mathrm{~K}, \quad \Theta_{y}=21.007 \mathrm{~K}, \quad \text { and } \\
\Theta_{z}=13.708 \mathrm{~K}, & \\
\mathrm{SO}_{2}: \Theta_{x}= & 2.9176 \mathrm{~K}, \quad \Theta_{y}=0.4952 \mathrm{~K}, \quad \text { and } \\
\Theta_{z} & =0.4234 \mathrm{~K} .
\end{aligned}
$$

The symmetry number $\sigma$ (number of equivalent conformations), which is 2, does not play a role here. See Table I for some results. For water, which is a relatively light molecule, we find after applying the first quantum correction the following errors: for $50 \mathrm{~K} 0.94 \%$, for $100 \mathrm{~K} 0.24 \%$, and for $500 \mathrm{~K} 0.01 \%$. For the much heavier molecule $\mathrm{SO}_{2}$ the error is (within four digit accuracy) zero for the four temperatures listed. That is, the first quantum correction gives essentially the exact result. It is important for the present development that the classical approximation to $\left\langle\left\langle H_{\text {rot }}\right\rangle\right\rangle$ [and accordingly also the left-hand side of Eq. (14)] is very good and that neglect of $F_{x}$ and $F_{y}$ does not introduce any error (within four decimal digits), while neglect of $F_{z}$ introduces a small error $(6 \%$ at $50 \mathrm{~K})$ for $\mathrm{H}_{2} \mathrm{O}$ and hardly any $(0.2 \%$ at $50 \mathrm{~K})$ for $\mathrm{SO}_{2}$.

Finally, combining Eqs. (5) and (10), we find an expression for the total one-rotor Slater sum $W_{1}$ that is very important for the development in Sec. IV, 
TABLE I. Approximate quantum correction to $\left\langle\left\langle H_{\text {rot }}\right\rangle\right\rangle /\left(k_{B} T\right)$ in column labeled "Approx," see Eq. (16) for its definition. See Eq. (15) for the definition of $F_{x}, F_{y}$, and $F_{z} . T$ is in kelvin. Column labeled "Exact" gives exact values of $\left\langle\left\langle H_{\text {rot }}\right\rangle\right\rangle /\left(k_{B} T\right)$ obtained from converged numerical calculations.

\begin{tabular}{|c|c|c|c|c|c|}
\hline$T$ & $F_{x}$ & $F_{y}$ & $F_{z}$ & Approx. & Exact \\
\hline \multicolumn{6}{|c|}{$\mathrm{H}_{2} \mathrm{O}$} \\
\hline 50 & 0.0000 & 0.0000 & 0.0837 & 1.4163 & 1.4031 \\
\hline 100 & 0.0000 & 0.0000 & 0.0437 & 1.4563 & 1.4528 \\
\hline 500 & 0.0000 & 0.0000 & 0.0091 & 1.4909 & 1.4908 \\
\hline 1000 & 0.0000 & 0.0000 & 0.0045 & 1.4955 & 1.4954 \\
\hline \multicolumn{6}{|c|}{$\mathrm{SO}_{2}$} \\
\hline 50 & 0.0000 & 0.0000 & 0.0028 & 1.4972 & 1.4972 \\
\hline 100 & 0.0000 & 0.0000 & 0.0014 & 1.4986 & 1.4986 \\
\hline 500 & 0.0000 & 0.0000 & 0.0003 & 1.4997 & 1.4997 \\
\hline 1000 & 0.0000 & 0.0000 & 0.0001 & 1.4999 & 1.4999 \\
\hline
\end{tabular}

$$
W_{1} \equiv\left\langle\mathbf{x}\left|e^{-\beta\left(H_{\mathrm{tr}}+H_{\mathrm{rot}}\right)}\right| \mathbf{x}\right\rangle=\frac{Q_{1}^{\mathrm{tr}}}{V} \frac{Q_{1}^{\mathrm{rot}}}{8 \pi^{2}}=\frac{Q_{1}}{V 8 \pi^{2}} .
$$

\section{THE EXPANSION OF $e^{-\beta\left(H_{0}+U\right)}$}

To simplify and unify notation we define

$$
\nabla_{a}(i) \equiv\left\{\begin{array}{cc}
(2 M)^{-1 / 2} p_{a}(i) & \text { for } a=1,2,3, \\
\left(2 I_{a-3}\right)^{-1 / 2} \mathcal{J}_{a-3}(i) & \text { for } a=4,5,6 .
\end{array}\right.
$$

The operator $\nabla_{a}(i)$ is Hermitian. The pair potential is $U\left(\mathbf{x}_{1}, \mathbf{x}_{2}\right)$ and the two-body Hamiltonian becomes $H=H_{0}$ $+U$, with $H_{0}=\Sigma_{i}\left[H_{\mathrm{tr}}(i)+H_{\mathrm{rot}}(i)\right]$. We will write

$$
H_{0}=\nabla_{\alpha} \nabla_{\alpha} \equiv \sum_{\alpha=1}^{12} \nabla_{\alpha} \nabla_{\alpha} \equiv \sum_{i=1}^{2} \sum_{a=1}^{6} \nabla_{a}(i) \nabla_{a}(i),
$$

where a sum over two particles and six components per particle is implied for two repeated Greek indices. Further, we write

$$
\widetilde{U}=\beta U
$$

and

$$
\widetilde{H}_{0}=\widetilde{\nabla}_{\alpha} \widetilde{\nabla}_{\alpha}
$$

with

$$
\widetilde{\nabla}_{\alpha}=\sqrt{\beta} \nabla_{\alpha} .
$$

Note that $\widetilde{U}$ and $\widetilde{H}_{0}$ are dimensionless, as is $\widetilde{\nabla}_{\alpha}$. Define, following Friedmann, ${ }^{3}$ the operator $T$ by

$$
T \equiv e^{\tilde{U}} e^{-\tilde{U}-\tilde{H}_{0}} e^{\tilde{H}_{0}} \Leftrightarrow e^{-\beta\left(H_{0}+U\right)}=e^{-\beta U} T e^{-\beta H_{0}}
$$

and expand it

$$
T=1+T^{(1)}+T^{(2)}+\cdots,
$$

where $T^{(k)}$ is of order $\widetilde{H}_{0}^{k}$, i.e., of order $\widetilde{\nabla}_{\alpha}^{2 k}$. We will give expressions for $T^{(1)}$ and $T^{(2)}$ in terms of $(\operatorname{ad} \widetilde{U})^{k} \widetilde{H}_{0}$, where

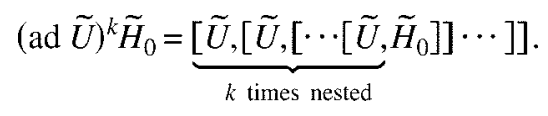

We define first and second derivatives

$$
\tilde{U}_{\alpha}=\left[\widetilde{\nabla}_{\alpha}, \tilde{U}\right]
$$

and

$$
\tilde{U}_{\alpha \alpha}=\left[\widetilde{\nabla}_{\alpha}, \tilde{U}_{\alpha}\right] .
$$

It is easy to show that

$$
\operatorname{ad} \tilde{U} \widetilde{H}_{0}=\widetilde{\nabla}_{\alpha}\left(\operatorname{ad} \tilde{U} \widetilde{\nabla}_{\alpha}\right)+\left(\operatorname{ad} \tilde{U} \widetilde{\nabla}_{\alpha}\right) \widetilde{\nabla}_{\alpha}=-\widetilde{U}_{\alpha \alpha}-2 \widetilde{U}_{\alpha} \widetilde{\nabla}_{\alpha},
$$

and, by applying ad $\widetilde{U}$ again,

$$
(\operatorname{ad} \tilde{U})^{2} \widetilde{H}_{0}=2\left(U_{\alpha}\right)^{2}, \quad(\operatorname{ad} \tilde{U})^{3} \widetilde{H}_{0}=0 .
$$

The latter property restricts expansions containing $(\operatorname{ad} \widetilde{U})^{k} \widetilde{H}_{0}$ to $k=2$.

Consider now

$$
V(t) \equiv e^{-t\left(\tilde{U}+\tilde{H}_{0}\right)}=e^{-t \tilde{U}} T(t) e^{-t \tilde{H}_{0}}
$$

and note that for $t=1$ we get the relation of Eq. (19), i.e., $T(1)$ is the operator we wish to expand, while $T(0)=1$. Differentiation gives

$$
\frac{d V}{d t}=-\tilde{U} e^{-t \tilde{U}} T(t) e^{-t \tilde{H}_{0}}-e^{-t \tilde{U}} T(t) e^{-t \tilde{H}_{0}} \widetilde{H}_{0}+e^{-t \tilde{U}} \frac{d T}{d t} e^{-t \tilde{H}_{0}} .
$$

Multiplication by $\exp (t \tilde{U})$ on the left and $\exp \left(t \widetilde{H}_{0}\right)$ on the right gives

$$
\frac{d T}{d t}=T(t) \widetilde{H}_{0}-e^{t \tilde{U}} \widetilde{H}_{0} e^{-t \tilde{U}} T(t) .
$$

Introduce the following commutator expansion: ${ }^{14}$

$$
e^{t \tilde{U}} \widetilde{H}_{0} e^{-t \tilde{U}}=\left(e^{t \operatorname{ad} \tilde{U}}\right) \widetilde{H}_{0}=\sum_{k=0}^{\infty} \frac{t^{k}}{k !}(\operatorname{ad} \tilde{U})^{k} \widetilde{H}_{0}
$$

so that 


$$
\frac{d T(t)}{d t}=\left[T(t), \widetilde{H}_{0}\right]-\left\{\sum_{k=1}^{2} \frac{t^{k}}{k !}(\operatorname{ad} \widetilde{U})^{k} \widetilde{H}_{0}\right\} T(t),
$$

where the summation ends at $k=2$, as we saw above, because $\widetilde{H}_{0}$ is a second-order differential operator. The terms in the summation are given by Eqs. (20) and (21). We solve this equation by iteration and the master recursion relation becomes

$$
\frac{d T^{(n+1)}}{d t}=\left[T^{(n)}(t), \tilde{H}_{0}\right]+\left[t\left(\widetilde{U}_{\alpha \alpha}+2 \tilde{U}_{\alpha} \nabla_{\alpha}\right)-t^{2} \widetilde{U}_{\alpha}^{2}\right] T^{(n)},
$$

with $T^{(0)}=1$.

Integration over $t^{\prime}$ from 0 to $t$ yields the expansion

$$
T^{(1)}(t)=-\sum_{k=1}^{2} \frac{t^{k+1}}{(k+1) !}(\operatorname{ad} \tilde{U})^{k} \tilde{H}_{0} .
$$

Hence $T^{(1)}(1)$ becomes

$$
T^{(1)}=\frac{1}{2} \widetilde{U}_{\alpha \alpha}+\widetilde{U}_{\alpha} \widetilde{\nabla}_{\alpha}-\frac{1}{3} \widetilde{U}_{\alpha}^{2} .
$$

Substitution of Eq. (25) into Eq. (23) and integration gives

$$
\begin{aligned}
T^{(2)}(t)= & -\sum_{k=1}^{2} \frac{t^{k+2}}{(k+2) !}\left[(\operatorname{ad} \tilde{U})^{k} \widetilde{H}_{0}, \widetilde{H}_{0}\right] \\
& +\sum_{k_{1}, k_{2}=1}^{2} \frac{t^{k_{1}+k_{2}+2}}{\left(k_{1}+k_{2}+2\right) k_{1} !\left(k_{2}+1\right) !}(\operatorname{ad} \tilde{U})^{k_{1}} \\
& \times \widetilde{H}_{0}(\operatorname{ad} \tilde{U})^{k_{2}} \widetilde{H}_{0} .
\end{aligned}
$$

This expansion gives $T^{(2)}$ after substitution $t=1$. After some straightforward but tedious algebra we find that the operator $T^{(2)}$ can be determined to be

$$
\begin{aligned}
T^{(2)}= & -\frac{1}{6} \widetilde{U}_{\alpha \alpha \beta \beta}+\frac{1}{8} \widetilde{U}_{\alpha \alpha} \widetilde{U}_{\beta \beta}+\frac{1}{6} \widetilde{U}_{\alpha \beta} \widetilde{U}_{\alpha \beta}+\frac{5}{12} \widetilde{U}_{\alpha} \widetilde{U}_{\alpha \beta \beta} \\
& -\frac{1}{6} \widetilde{U}_{\alpha} \widetilde{U}_{\alpha} \widetilde{U}_{\beta \beta}-\frac{4}{15} \widetilde{U}_{\alpha} \widetilde{U}_{\beta} \widetilde{U}_{\alpha \beta}+\frac{1}{18} \widetilde{U}_{\alpha} \widetilde{U}_{\alpha} \widetilde{U}_{\beta} \widetilde{U}_{\beta} \\
& -\frac{2}{3} \widetilde{U}_{\alpha \alpha \beta} \widetilde{\nabla}_{\beta}+\frac{1}{2} \widetilde{U}_{\alpha \alpha} \widetilde{U}_{\beta} \widetilde{\nabla}_{\beta}+\frac{5}{6} \widetilde{U}_{\alpha} \widetilde{U}_{\alpha \beta} \widetilde{\nabla}_{\beta} \\
& -\frac{1}{3} \widetilde{U}_{\alpha} \widetilde{U}_{\alpha} \widetilde{U}_{\beta} \widetilde{\nabla}_{\beta}-\frac{2}{3} \widetilde{U}_{\alpha \beta} \widetilde{\nabla}_{\alpha} \widetilde{\nabla}_{\beta}+\frac{1}{2} \widetilde{U}_{\alpha} \widetilde{U}_{\beta} \widetilde{\nabla}_{\alpha} \widetilde{\nabla}_{\beta} \\
& -\frac{1}{3} \widetilde{U}_{\alpha}\left[\widetilde{\nabla}_{\beta}^{2}, \widetilde{\nabla}_{\alpha}\right] .
\end{aligned}
$$

At this point we can show that only $T^{(1)}$ and $T^{(2)}$ contribute to the first quantum correction of $B_{2}(T)$. The quantum corrections due to $T^{(k)}$ contain by definition $(2 k)$ th derivatives. We see in Eq. (26) that the terms appearing for $k=1$ are multiples of $\left(\widetilde{\nabla}_{\alpha} \widetilde{\nabla}_{\alpha} \widetilde{U}\right)$ and $\left(\widetilde{\nabla}_{\alpha} \widetilde{U}\right)\left(\widetilde{\nabla}_{\alpha} \widetilde{U}\right)$, but also that a term $\left(\widetilde{\nabla}_{\alpha} \widetilde{U}\right) \widetilde{\nabla}_{\alpha}$ with trailing operator $\widetilde{\nabla}_{\alpha}$ appears. In general the $T^{(n)}$ terms have trailing polynomials $P(\widetilde{\boldsymbol{\nabla}})$ of different order and we will see below that these arise in $\langle\mathbf{x}| P(\widetilde{\boldsymbol{\nabla}})$ $\times \exp \left(-\widetilde{H}_{0}\right)|\mathbf{x}\rangle$. We discussed in the preceding section that many such expectation values vanish because of time reversal or $D_{2}$ symmetry and so does the one with $P(\tilde{\nabla}) \equiv \widetilde{\nabla}_{\alpha}$. Consequently, the term with trailing $\widetilde{\nabla}_{\alpha}$ in $T^{(1)}$ does not contribute to $Q_{2}$. The remaining terms in $T^{(1)}$ do contribute to $\hbar^{2}$ terms.
The terms of $T^{(2)}$ are by definition fourth order in the components of $\tilde{\boldsymbol{\nabla}}$. In Eq. (28) we see $\hbar^{4}$ terms of the type

$$
\left(\widetilde{\nabla}_{\alpha} \widetilde{U}\right)\left(\widetilde{\nabla}_{\alpha} \widetilde{U}\right)\left(\widetilde{\nabla}_{\beta} \widetilde{\nabla}_{\beta} \widetilde{U}\right), \quad\left(\nabla_{\alpha} \nabla_{\alpha} \nabla_{\beta} \nabla_{\beta} \widetilde{U}\right) \text { etc. }
$$

and also terms with trailing operators. At first sight it seems as if $T^{(2)}$ is of highest order 3 in the trailing operators, because of the presence of $\left[\tilde{\nabla}_{\beta}^{2}, \widetilde{\nabla}_{\alpha}\right]$. The linear momenta commute with themselves and also with the angular momenta, so that only the case $\widetilde{\nabla}_{\beta}^{2} \propto \mathcal{J}_{b}^{2}$ and $\tilde{\nabla}_{\alpha} \propto \mathcal{J}_{a}$ is of concern. In general, the commutation relations of $\mathcal{J}_{a}$ 's imply that the commutator $\left[\mathcal{J}_{b}^{2}, \mathcal{J}_{a_{1}} \mathcal{J}_{a_{2}} \cdots \mathcal{J}_{a_{n}}\right]$ is of order $n+1$ in the operators $\mathcal{J}_{x}, \mathcal{J}_{y}$, and $\mathcal{J}_{z}$ so that $T^{n}$ is of maximum order 2 in the trailing operators.

Noting that $\left\langle\mathbf{x}\left|\left[\tilde{\nabla}_{\beta}^{2}, \tilde{\nabla}_{\alpha}\right] \exp \left(-\widetilde{H}_{0}\right)\right| \mathbf{x}\right\rangle$ vanishes, as do the cross terms $\widetilde{\nabla}_{\alpha} \widetilde{\nabla}_{\beta}$, we see that $T^{(2)}$ contributes only terms of the type $\left(\widetilde{\nabla}_{\alpha} \widetilde{\nabla}_{\alpha} \widetilde{U}\right)$ and $\left(\widetilde{\nabla}_{\alpha} \widetilde{U}\right)\left(\widetilde{\nabla}_{\alpha} \widetilde{U}\right)$ to the first quantum correction, together with the $O\left(\hbar^{0}\right)$ term of $\langle\boldsymbol{\omega}| \mathcal{J}_{a}^{2}$ $\times \exp \left(-\widetilde{H}_{0}\right)|\boldsymbol{\omega}\rangle$. The first-order $\left[O\left(\hbar^{2}\right)\right]$ term in the latter expectation value is given in Eq. (14). Obviously this term contributes $O\left(\hbar^{4}\right)$ to $B_{2}(T)$ and can consistently be neglected. (In the preceding section the size of this term was discussed for $\mathrm{H}_{2} \mathrm{O}$ and $\mathrm{SO}_{2}$ as examples of asymmetric top molecules.) In conclusion, in order to compute the first-order quantum correction we need at least $T^{(1)}$ and $T^{(2)}$.

By the principle of mathematical induction we show that $T^{(n+1)}$ is of maximum order $n+1$ in the trailing operators. Assume to that end that the trailing polynomial of $T^{(n)}$ is of maximum order $n$ in the components of $\widetilde{\nabla}$. We have seen that this is true for $n=0,1$, and 2. From the master relation [Eq. (24)] follows that $d T^{(n+1)} / d t$ is of order $n+1$ in the $\tilde{\nabla}_{\alpha}$ 's, since we have just argued that $\left[T^{(n)}(t), \widetilde{H}_{0}\right]$ is of order $n+1$. Furthermore, integration does not change this order, and so $T^{(n+1)}$ is of maximum order $n+1$ in the components of $\widetilde{\boldsymbol{\nabla}}$. For $T^{(3)}$ this implies that the maximum order of its trailing operator is 3 , so that the derivatives of the potential are of minimum order 3 and contribute to $\hbar^{3}$ terms. By the same argument the elements $T^{(k)}$ for $k>3$ do not give $\hbar^{2}$ contributions, which is why only $T^{(1)}$ and $T^{(2)}$ contribute in order $\hbar^{2}$.

\section{CONTRIBUTIONS TO $B_{2}$}

In this section the classical and first quantum contribution to the second virial coefficient $B_{2}(T)$ will be given. We reiterate that only $Q_{2}$ is needed. Using the general expression for $Q_{N}$ valid in the Boltzmann limit (no exchange), we write

$$
\begin{aligned}
Q_{2} & =\frac{1}{2 !} \int\left\langle\mathbf{x}_{1}, \mathbf{x}_{2}\left|e^{-\beta H}\right| \mathbf{x}_{1}, \mathbf{x}_{2}\right\rangle d \mathbf{x}_{1} d \mathbf{x}_{2} \\
& =\frac{1}{2 !} \int e^{-\beta U\left(\mathbf{x}_{1}, \mathbf{x}_{2}\right)}\left\langle\mathbf{x}_{1}, \mathbf{x}_{2}\left|T e^{-\beta H_{0}}\right| \mathbf{x}_{1}, \mathbf{x}_{2}\right\rangle d \mathbf{x}_{1} d \mathbf{x}_{2} .
\end{aligned}
$$

Since $U$ is a local function of $\mathbf{x}_{1}$ and $\mathbf{x}_{2}$ we were able to use here

$$
\left\langle\mathbf{x}_{1}, \mathbf{x}_{2}\left|e^{-\beta U}\right| \mathbf{x}_{1}^{\prime}, \mathbf{x}_{2}^{\prime}\right\rangle=\delta\left(\mathbf{x}_{1}^{\prime}-\mathbf{x}_{1}\right) \delta\left(\mathbf{x}_{2}^{\prime}-\mathbf{x}_{2}\right) e^{-\beta U\left(\mathbf{x}_{1}, \mathbf{x}_{2}\right)} .
$$




\section{A. Contributions from $T^{(0)}$}

Insert $T^{(0)}=1$ and twice the Slater sum [Eq. (17)] into Eq. (29) and we get the classical contribution to the twobody partition function,

$$
Q_{2}^{(0)}=\frac{1}{2 !}\left(\frac{Q_{1}}{V 8 \pi^{2}}\right)^{2} \int d \mathbf{x}_{1} d \mathbf{x}_{2} e^{-\beta U\left(\mathbf{x}_{1}, \mathbf{x}_{2}\right)} .
$$

Using Eq. (2) it follows that the classical contribution to $B_{2}$ for a gas consisting of interacting asymmetric tops is

$$
B_{2}^{(0)}=\frac{1}{V 128 \pi^{4}} \int\left[1-e^{-\beta U\left(\mathbf{x}_{1}, \mathbf{x}_{2}\right)}\right] d \mathbf{x}_{1} d \mathbf{x}_{2} .
$$

\section{B. Contributions from $T^{(1)}$}

From Eq. (26) we get the contribution $Q_{2}^{(1)}$ to $Q_{2}$ arising from $T^{(1)}$

$$
\begin{aligned}
Q_{2}^{(1)}= & \frac{1}{2 !} \int e^{-\beta U\left(\mathbf{x}_{1}, \mathbf{x}_{2}\right)}\left[\frac{1}{2} \widetilde{U}_{\alpha \alpha}\left(\mathbf{x}_{1}, \mathbf{x}_{2}\right)-\frac{1}{3} \widetilde{U}_{\alpha}\left(\mathbf{x}_{1}, \mathbf{x}_{2}\right)^{2}\right] \\
& \times\left\langle\mathbf{x}_{1}, \mathbf{x}_{2}\left|e^{-\tilde{H}_{0}}\right| \mathbf{x}_{1}, \mathbf{x}_{2}\right\rangle d \mathbf{x}_{1} d \mathbf{x}_{2} .
\end{aligned}
$$

We used here that $\widetilde{U}_{\alpha \alpha}$ and $\widetilde{U}_{\alpha}^{2}$ are local and hence diagonal in the $\mathbf{x}$ representation. We dropped the term with trailing $\widetilde{\nabla}_{\alpha}$, because it gives a vanishing contribution. Using Eq. (17) for the Slater sum gives

$$
\begin{aligned}
Q_{2}^{(1)}= & \left(\frac{Q_{1}}{V 8 \pi^{2}}\right)^{2} \int e^{-\beta U\left(\mathbf{x}_{1}, \mathbf{x}_{2}\right)}\left[\frac{1}{4} \widetilde{U}_{\alpha \alpha}\left(\mathbf{x}_{1}, \mathbf{x}_{2}\right)\right. \\
& \left.-\frac{1}{6} \widetilde{U}_{\alpha}\left(\mathbf{x}_{1}, \mathbf{x}_{2}\right)^{2}\right] d \mathbf{x}_{1} d \mathbf{x}_{2} .
\end{aligned}
$$

Knowing that $\widetilde{\nabla}_{\alpha}$ is Hermitian and purely imaginary, we can rewrite the second derivative as a product of two first derivatives, namely,

$$
\begin{aligned}
\int e^{-\tilde{U}} \widetilde{\nabla}_{\alpha} \widetilde{\nabla}_{\alpha} \tilde{U} d \mathbf{x}_{1} d \mathbf{x}_{2} & =\int\left(\widetilde{\nabla}_{\alpha} e^{-\tilde{U}}\right)^{*}\left(\widetilde{\nabla} \widetilde{U}_{\alpha}\right) d \mathbf{x}_{1} d \mathbf{x}_{2} \\
& =-\int\left(e^{-\tilde{U}} \widetilde{\nabla}_{\alpha} \widetilde{U}\right)^{*}\left(\widetilde{\nabla}_{\alpha} \widetilde{U}\right) d \mathbf{x}_{1} d \mathbf{x}_{2} \\
& =\int e^{-\tilde{U}} \widetilde{U}_{\alpha} \widetilde{U}_{\alpha} d \mathbf{x}_{1} d \mathbf{x}_{2} .
\end{aligned}
$$

This result may be summarized by the effective [valid inside the integral with kernel $\exp (-\widetilde{U})]$ equation

$$
H_{0} U \equiv \nabla_{\alpha} \nabla_{\alpha} \stackrel{\text { eff }}{=}=\beta\left(\nabla_{\alpha} U\right)\left(\nabla_{\alpha} U\right) .
$$

Using this in Eq. (32), we get the final contribution to $Q_{2}^{(1)}$ from $T^{(1)}$

$$
Q_{2}^{(1)}=\frac{1}{12}\left(\frac{Q_{1}}{V 8 \pi^{2}}\right)^{2} \int e^{-\tilde{U}\left(\mathbf{x}_{1} \mathbf{x}_{2}\right)}\left[\tilde{U}_{\alpha}\left(\mathbf{x}_{1} \mathbf{x}_{2}\right)\right]^{2} d \mathbf{x}_{1} d \mathbf{x}_{2} .
$$

Anticipating that the contribution from $T^{(2)}$ has the same form, the adding of this result to $B_{2}^{(0)}$ will be postponed to the last section.

\section{Contributions from $T^{(2)}$}

As discussed in the preceding section, only the second derivatives of the potential arising in Eq. (28) need be considered in the $\hbar^{2}$ approximation. These terms contain trailing polynomials bilinear in $\tilde{\nabla}_{\alpha} \tilde{\nabla}_{\beta}$. Terms of the type $\nabla_{a}(i) \nabla_{b}(j)$ with different particle index $i \neq j$ vanish. For equal particle index only the diagonal terms $\widetilde{\nabla}_{\alpha} \widetilde{\nabla}_{\alpha}$ survive; this is true for the translational as well as the rotational derivatives. Hence $T^{(2)}$ contributes:

$$
\begin{aligned}
Q_{2}^{(2)}= & \frac{1}{2} \int e^{-\tilde{U}\left(\mathbf{x}_{1} \mathbf{x}_{2}\right)}\left(\frac{1}{2} \tilde{U}_{\alpha}\left(\mathbf{x}_{1} \mathbf{x}_{2}\right)^{2}-\frac{2}{3} \widetilde{U}_{\alpha \alpha}\left(\mathbf{x}_{1} \mathbf{x}_{2}\right)\right) \\
& \times\left\langle\mathbf{x}_{1} \mathbf{x}_{2}\left|\widetilde{\nabla}_{\alpha} \widetilde{\nabla}_{\alpha} e^{-\tilde{H}_{0}}\right| \mathbf{x}_{1} \mathbf{x}_{2}\right\rangle d \mathbf{x}_{1} d \mathbf{x}_{2} .
\end{aligned}
$$

Recalling that the summation convention is employed (sum over two particles and six degrees of freedom per particle), it is easily shown that for the second derivatives of an arbitrary function $V\left(\mathbf{x}_{1}, \mathbf{x}_{2}\right)$

$$
\widetilde{V}_{\alpha \alpha}\left\langle\mathbf{x}_{1} \mathbf{x}_{2}\left|\widetilde{\nabla}_{\alpha} \widetilde{\nabla}_{\alpha} e^{-\tilde{H}_{0}}\right| \mathbf{x}_{1} \mathbf{x}_{2}\right\rangle=\frac{1}{2}\left(\frac{Q_{1}}{V 8 \pi^{2}}\right)^{2} \widetilde{V}_{\alpha \alpha}
$$

holds. Note that this is equally valid for translational [cf. Eq. (7)] as well as rotational [with neglect of $F_{\alpha}$, cf. Eq. (14)] derivatives. Hence

$$
\begin{aligned}
Q_{2}^{(2)}= & \left(\frac{Q_{1}}{V 8 \pi^{2}}\right)^{2} \int e^{-\tilde{U}\left(\mathbf{x}_{1} \mathbf{x}_{2}\right)}\left(\frac{1}{8} \widetilde{U}_{\alpha}\left(\mathbf{x}_{1} \mathbf{x}_{2}\right)^{2}\right. \\
& \left.-\frac{1}{6} \widetilde{U}_{\alpha \alpha}\left(\mathbf{x}_{1} \mathbf{x}_{2}\right)\right) d \mathbf{x}_{1} d \mathbf{x}_{2} \\
= & -\frac{1}{24}\left(\frac{Q_{1}}{V 8 \pi^{2}}\right)^{2} \int e^{-\tilde{U}\left(\mathbf{x}_{1} \mathbf{x}_{2}\right)} \widetilde{U}_{\alpha}\left(\mathbf{x}_{1} \mathbf{x}_{2}\right)^{2} d \mathbf{x}_{1} d \mathbf{x}_{2},
\end{aligned}
$$

where for the second equality Eq. (33) has been employed. Adding this expressions to Eq. (34) and reintroducing summations give finally the total correction of order $\hbar^{2}$ to the partition function $Q_{2}$ as

$$
\begin{aligned}
Q_{2}^{(1+2)}= & \frac{1}{24}\left(\frac{Q_{1}}{V 8 \pi^{2}}\right)^{2} \int e^{-\tilde{U}\left(\mathbf{x}_{1} \mathbf{x}_{2}\right)} \\
& \times \sum_{i=1}^{2} \sum_{a=1}^{6}\left[\widetilde{\nabla}_{a}(i) \tilde{U}\left(\mathbf{x}_{1} \mathbf{x}_{2}\right)\right]^{2} d \mathbf{x}_{1} d \mathbf{x}_{2} .
\end{aligned}
$$

Upon recalling the definition of $\nabla_{a}(i)$ from Eq. (18), we have a result for asymmetric rotors that is the same as that obtained earlier by Friedmann for symmetric rotors (two equal inertia moments).

\section{Virial coefficient $\boldsymbol{B}_{2}$}

If we now add the classical contribution [Eq. (30)] to $Q_{2}^{(1+2)}$ [Eq. (37)] and use this in Eq. (2), we obtain the second virial coefficient correct through $\hbar^{2}$, namely, 


$$
\begin{aligned}
B_{2}= & \frac{1}{128 V \pi^{4}} \int\left[1-e^{-\widetilde{U}\left(\mathbf{x}_{1} \mathbf{x}_{2}\right)}\right. \\
& \left.\times\left(1+\frac{1}{12} \sum_{i=1}^{2} \sum_{a=1}^{6}\left[\tilde{\nabla}_{a}(i) \tilde{U}\left(\mathbf{x}_{1} \mathbf{x}_{2}\right)\right]^{2}\right)\right] d \mathbf{x}_{1} d \mathbf{x}_{2} .
\end{aligned}
$$

Using Eq. (33) we can rewrite this equation thus

$$
\begin{aligned}
B_{2}(T)= & \frac{1}{128 V \pi^{4}} \int \cdots \int\left\{1-e^{-\beta U\left(\mathbf{x}_{1} \mathbf{x}_{2}\right)}\right. \\
& \left.\times\left[1+\frac{\beta^{2}}{12} H_{0}(U)\right]\right\} d \mathbf{x}_{1} d \mathbf{x}_{2},
\end{aligned}
$$

which has precisely the same form as the equation previously derived by T Pack ${ }^{2}$ for a gas of symmetric rotors. (Note that T Pack defines $B_{2}$ as a molar property, whereas here it is a molecular property, the difference being a factor $N_{A}$, which is Avogadro's number.)

To simplify the integrals somewhat, we introduce

$$
\mathbf{R}=\left(\mathbf{r}_{1}+\mathbf{r}_{1}\right) / 2
$$

and

$$
\mathbf{r}=\mathbf{r}_{1}-\mathbf{r}_{2}
$$

and assume that $U\left(\mathbf{r}_{1}, \mathbf{r}_{2}, \boldsymbol{\omega}_{1}, \boldsymbol{\omega}_{2}\right)$ does not depend on the position $\mathbf{R}$ of the dimer mass center, but only on the distance $\mathbf{r}$ and $\boldsymbol{\omega}_{1}, \boldsymbol{\omega}_{2}$. Use

$$
d \mathbf{r}_{1} d \mathbf{r}_{2}=d \mathbf{R} d \mathbf{r}
$$

and

$$
\int d \mathbf{R}=V
$$

Let $\mathbf{P}$ and $\mathbf{p}$ be conjugate to $\mathbf{R}$ and $\mathbf{r}$, then it is well known that

$$
\frac{p(1)^{2}}{2 M}+\frac{p(2)^{2}}{2 M}=\frac{P^{2}}{4 M}+\frac{p^{2}}{M}
$$

where $\mathbf{P}$ and $\mathbf{p}$ are the linear momentum of the dimer center of mass and the dimer relative particle (with reduced mass $M / 2$ ), respectively. Noting that $P^{2} U=0$ we obtain finally an expression for $B_{2}(T)$ correct through $\hbar^{2}$,

$$
\begin{aligned}
B_{2}(T)= & \frac{1}{128 \pi^{4}} \int\left[1-e^{-\tilde{U}\left(\mathbf{r}, \boldsymbol{\omega}_{1}, \boldsymbol{\omega}_{2}\right)}\right. \\
& \times\left(1+\frac{\beta}{12 M} \sum_{a=1}^{3}\left[p_{a} \tilde{U}\left(\mathbf{r}, \boldsymbol{\omega}_{1}, \boldsymbol{\omega}_{2}\right)\right]^{2}\right. \\
& \left.+\frac{1}{12} \sum_{i=1}^{2} \sum_{a=4}^{6}\left[\widetilde{\nabla}_{a}(i) \widetilde{U}\left(\mathbf{r}, \boldsymbol{\omega}_{1}, \boldsymbol{\omega}_{2}\right)\right]^{2}\right] d \mathbf{r} d \boldsymbol{\omega}_{1} d \boldsymbol{\omega}_{2} .
\end{aligned}
$$

The quantum correction contains an inner product of forces,

$$
\frac{\beta}{12 M} \sum_{a=1}^{3}\left[p_{a} \tilde{U}\left(\mathbf{r}, \boldsymbol{\omega}_{1}, \boldsymbol{\omega}_{2}\right)\right]^{2}=-\frac{\hbar^{2} \beta^{3}}{M} \sum_{a=x, y, z}\left(\frac{\partial U}{\partial r_{a}}\right)^{2}
$$

and of torques,

$$
\sum_{i=1}^{2} \sum_{a=4}^{6}\left[\widetilde{\nabla}_{a}(i) \tilde{U}\left(\mathbf{r}, \boldsymbol{\omega}_{1}, \boldsymbol{\omega}_{2}\right)\right]^{2}=\beta^{3} \sum_{i=1}^{2} \sum_{a=x, y, z} \frac{1}{2 I_{a}}\left[\mathcal{J}_{a}(i) U\right]^{2} .
$$

Both contributions are dimensionless. The quantum correction is purely dynamic as it does not contain kinetic energy terms.

\section{SUMMARY}

We have drastically simplified Friedmann's approach to the quantum correction of canonical partition functions of symmetric tops. This was achieved by considering bodyfixed angular momenta instead of derivatives with respect to Euler angles. The simplification reduced the length of the derivations considerably and it gives as an extra bonus the first quantum correction for asymmetric tops. The partition function was applied in the derivation of the second virial coefficient $B_{2}$. The virial coefficient obtained has exactly the same form as the one published by $\mathrm{T}$ Pack, ${ }^{2}$ but without the restriction of its applicability to symmetric tops only. For further discussion and application of the formula for $B_{2}$ to actual systems we refer to T Pack's paper. Suffice it to say that the final equation is most easily applied when the intermolecular potential $U\left(\mathbf{r}, \boldsymbol{\omega}_{1}, \boldsymbol{\omega}_{2}\right)$ is expanded in a product of $D$ matrices and spherical harmonics: $D_{m_{1} k_{1}}^{l_{1}}\left(\boldsymbol{\omega}_{1}\right) D_{m_{2} k_{2}}^{l_{2}}\left(\boldsymbol{\omega}_{2}\right) Y_{M}^{L}(\hat{\mathbf{r}})$, see e.g., Ref. 15. The action of $\mathcal{J}_{x}, \mathcal{J}_{y}$, and $\mathcal{J}_{z}$, is easy after expressing these operators (that act on the column indices of the $D$ matrices) in terms of shift operators. Expression of $\mathbf{p}$ in spherical polar coordinates makes the action on $Y_{M}^{L}(\hat{\mathbf{r}})$ also well defined and easy.

${ }^{1}$ R. Moszynski, T. Korona, T. G. A. Heijmen, P. E. S. Wormer, A. van der Avoird, and B. Schramm, Pol. J. Chem. 72, 1479 (1998).

${ }^{2}$ R. T Pack, J. Chem. Phys. 78, 7217 (1983).

${ }^{3}$ H. Friedmann, Adv. Chem. Phys. 4, 225 (1962).

${ }^{4}$ D. A. McQuarrie, Statistical Mechanics (Harper and Row, New York, 1976).

${ }^{5}$ E. Beltrami, Mem. Accad. Bologna 8, 551 (1868).

${ }^{6}$ B. Podolsky, Phys. Rev. 32, 812 (1928).

${ }^{7}$ H. Margenau and G. M. Murphy, The Mathematics of Physics and Chemistry (Van Nostrand, New York, 1956).

${ }^{8}$ L. C. Biedenharn and J. D. Louck, Angular Momentum in Quantum Physics, Encyclopedia of Mathematics Vol. 8 (Addison-Wesley, Reading, 1981).

${ }^{9}$ D. ter Haar, Elements of Statistical Mechanics (Holt, Rinehart, and Winston, New York, 1961).

${ }^{10}$ A. R. Edmonds, Angular Momentum in Quantum Mechanics, 2nd ed. (Princeton University Press, Princeton, 1960).

${ }^{11}$ R. S. Mulliken, Phys. Rev. 59, 873 (1941).

${ }^{12}$ H. W. Kroto, Molecular Rotation Spectra (Dover, New York, 1992).

${ }^{13}$ K. F. Stripp and J. G. Kirkwood, J. Chem. Phys. 19, 1131 (1951).

${ }^{14}$ M. Hausner and J. T. Schwartz, Lie Groups; Lie Algebras (Gordon and Breach, New York, 1968).

${ }^{15}$ A. van der Avoird, P. E. S. Wormer, F. Mulder, and R. M. Berns, Top. Curr. Chem. 93, 1 (1980). 